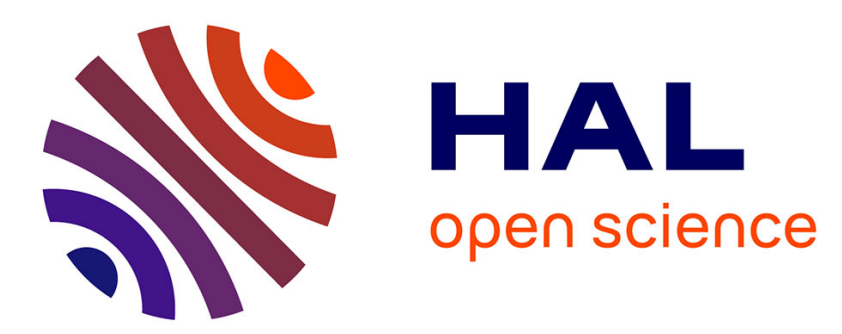

\title{
Analysis of artificial pressure equations in numerical simulations of a turbulent channel flow
}

Dorian Dupuy, Adrien Toutant, Françoise Bataille

\section{To cite this version:}

Dorian Dupuy, Adrien Toutant, Françoise Bataille. Analysis of artificial pressure equations in numerical simulations of a turbulent channel flow. Journal of Computational Physics, 2020, 411, pp.109407. 10.1016/j.jcp.2020.109407) . hal-02802118

\section{HAL Id: hal-02802118 \\ https://hal.science/hal-02802118}

Submitted on 5 Jun 2020

HAL is a multi-disciplinary open access archive for the deposit and dissemination of scientific research documents, whether they are published or not. The documents may come from teaching and research institutions in France or abroad, or from public or private research centers.
L'archive ouverte pluridisciplinaire HAL, est destinée au dépôt et à la diffusion de documents scientifiques de niveau recherche, publiés ou non, émanant des établissements d'enseignement et de recherche français ou étrangers, des laboratoires publics ou privés. 


\title{
Analysis of artificial pressure equations in numerical simulations of a turbulent channel flow
}

\author{
Dorian Dupuy ${ }^{1}$, Adrien Toutant*1, and Françoise Bataille ${ }^{1}$ \\ ${ }^{1}$ PROMES CNRS, Université de Perpignan Via Domitia, Rambla de la thermodynamique, \\ Tecnosud, 66100 Perpignan, France \\ (Published version: Journal of Computational Physics 411, 109407 (2020); \\ https://doi.org/10.1016/j.jcp.2020.109407)
}

\begin{abstract}
Recently, several methods have been proposed to simulate incompressible fluid flows using an artificial pressure evolution equation, avoiding the resolution of a Poisson equation. These methods can be seen as various levels of approximation of the compressible Navier-Stokes equation in the low Mach number limit. We study the simulation of incompressible wall-bounded flows using several artificial pressure equations in order to determine the most relevant approximations. The simulations are stable using a finite difference method in a staggered grid system, even without diffusive term, and converge to the incompressible solution, both in direct numerical simulations and for coarser meshes, to be used in large-eddy simulations. A pressure equation with a convective and a diffusive term produces a more accurate solution than a compressible solver or methods involving more approximations. This suggests that it is near to an optimal level of approximation. The presence of a convective term in the pressure evolution equation is in particular crucial for the accuracy of the method. The rate of convergence of the solution in terms of artificial Mach number is studied numerically and validates the theoretical quadratic convergence rate. We demonstrate that this property can be used to accelerate the rate of convergence using an extrapolation in terms of artificial Mach number. Since the approach is based on an explicit and local system of equations, the numerical procedure is massively parallelisable and has low memory requirements.
\end{abstract}

\section{Introduction}

Compressibility effects in fluids are characterised by the Mach number. When the Mach number tends towards zero, the velocity of acoustic waves becomes arbitrarily large compared to the velocity of the fluid, leading to the instantaneous propagation of any pressure disturbance. If conductive heat transfers and density variations are neglected, the Navier-Stokes equations converge in the low Mach number regime to the incompressible Navier-Stokes equations [49, 50, 2, 3], given for a Newtonian fluid by:

$$
\begin{gathered}
\frac{\partial U_{i}}{\partial t}=-\frac{\partial U_{j} U_{i}}{\partial x_{j}}-\frac{1}{\rho} \frac{\partial P}{\partial x_{i}}+\nu \frac{\partial^{2} U_{i}}{\partial x_{j} \partial x_{j}}, \\
\frac{\partial U_{j}}{\partial x_{j}}=0
\end{gathered}
$$

${ }^{*}$ Corresponding author : adrien.toutant@univ-perp.fr 
with $\rho$ the density, $\nu$ the kinematic viscosity, $t$ the time, $P$ the pressure, $U_{i}$ the $i$-th component of velocity and $x_{i}$ the Cartesian coordinate in $i$-th direction. The pressure in the incompressible Navier-Stokes equations acts as a Lagragian multiplier of the incompressibility constraint (2). Usually, the pressure is determined from the resolution of a Poisson equation together with a predictor-corrector projection scheme [13, 58]. This operation is costly and often represents the most computationally expensive part of the numerical resolution of the incompressible Navier-Stokes equations.

Numerical methods based on an explicit and local evolution equation for the pressure have been developed to avoid the resolution of a Poisson equation. However, the use of the Navier-Stokes equations in their compressible form is inefficient at low Mach number because of the large disparity between the velocity of the fluid and the speed of sound. In artificial compressibility approaches, this numerical issue is addressed through the artificial reduction of the velocity of acoustic waves. First proposed by Chorin [12], the artificial compressibility $(\mathrm{AC})$ method uses an explicit evolution equation for the pressure to enforce the incompressibility constraint

$$
\frac{\partial P}{\partial t}=-\rho c^{2} \frac{\partial U_{j}}{\partial x_{j}},
$$

with $c$ the artificial speed of sound. This pressure evolution equation drives the velocity towards a solenoidal field in the limit of steady state, as the time derivative of the pressure vanishes $[11,35,44,45]$. To extend the approach to unsteady flows, a dual timestepping procedure may be used. In that case, the system is for each physical timestep iterated in pseudo-time until convergence. This approach has been followed by various authors $[42$, $31,48,39,1,41,36]$. A review on the error analysis of artificial compressibility methods is given by Shen [57].

The use of the artificial compressibility method without subiteration has also been suggested in the literature for unsteady flows, as well as various related methods [52]. O'Rourke and Bracco [54] proposed a scaling called $\alpha$-transformation to artificially increase the effective Mach number. In the pressure gradient scaling (PGS) method [55], the pressure gradient in the momentum equation is modified to decrease the speed of sound. Wang and Trouvé [63] suggested the acoustic speed reduction (ASR) method, which keeps the momentum equation intact and instead alters the energy or pressure equation. A variant of the ASR method, the artificial acoustic stiffness reduction method (AASCM), was developed by Salinas-Vázquez et al. [56] to improve the stability of the numerical method. In Guerra and Gustafsson [22], Merkle and Choi [44, 45], a perturbation expansion procedure is used to obtain a system of equations valid at arbitrarily low Mach numbers, following mathematic studies of the convergence of the solution of the compressible Navier-Stokes equations to the solution of the incompressible Navier-Stokes equations [32, 33, 34]. Karlin et al. [30] recommended the use of the kinetically reduced local Navier-Stokes (KRLNS) equations [4] for the grand potential and the momentum. The entropically damped artificial compressibility (EDAC) method of Clausen [15] adds a diffusive term to the pressure evolution equation, and sometimes a convective term. Toutant [60] derived a general pressure (GP) equation from the compressible Navier-Stokes equation, which is equivalent. Tessarotto et al. [59] derived an exact pressure evolution equation for incompressible fluids based on the inverse kinetic theory approach proposed by Ellero and Tessarotto [21]. The lattice-Boltzmann (LB) method, which solves the Boltzmann transport equation on a discretised phase space [6], is also closely related to the AC method [28, 53, 5]. All these methods are explicit in time and local in space and thus particularly amenable to massively parallel GPU-based simulations and have low memory requirements $[24,25,26,29,41]$. 
The KRLNS equations [30, 9, 24, 25, 26], the EDAC method [15, 16, 29] and GP equation [61] have been applied for different viscous incompressible flows. Kajzer and Pozorski [29] used the EDAC method to perform direct numerical simulations of a turbulent channel flow at the friction Reynolds number 180 and 395 on a collocated grid system. Given the large number of artificial pressure equations suggested in the literature, the relevance of the various approximations made in each method is not clear. In this paper, some of these approximations are analysed numerically using successive simplifications of the compressible Navier-Stokes equations.

We consider the numerical simulation of a three-dimensional turbulent channel flow at a friction Reynolds number of 180 . First, we study the artificial compressibility method, investigate the effect of the artificial Mach number $(M a)$ on the accuracy of the simulation and show that the method is stable for all artificial Mach numbers investigated with the proposed numerical method. Then, we analyse the simulation of the channel with a variant of the artificial compressibility method which includes a convective term and a diffusive term in the pressure evolution equation, as in the GP equation [60] or as in the pressure equation of the EDAC method [15]. Two intermediate numerical methods are also considered in order to assess separately the effect of the convective term and of the diffusive term on the accuracy of the simulation. Finally, the method is compared to the simulation of the compressible Navier-Stokes equations at low Mach number, which is sometimes referred to as the weakly compressible (WC) method. The simulations are carried out on two meshes: a coarse mesh and a fine mesh, which is sufficiently resolved for mesh-converged direct numerical simulations. While previous authors [52, 15, 16, 29] favoured a collocated grid system, we use a finite difference method in a staggered grid system with a third-order Runge-Kutta time scheme. The accuracy of the simulations is examined by comparison of the results to simulations of the channel using a projection method for the same mesh, domain size and numerical schemes. The convergence in terms of artificial Mach number of the methods to the reference projection simulation is studied numerically. The extrapolation of two simulations with the same mesh at two different artificial Mach numbers, following the expected quadratic $\mathcal{O}\left(M a^{2}\right)$ rate of convergence of the solution $[14,34,52,53]$, can be used to accelerate the convergence and obtain a simulation with a smaller velocity divergence.

A derivation of the artificial pressure equations used in this paper is given in section 2 in order to clarify the differences between the selected methods. The channel flow configuration and the numerical method are presented in section 3 . The results are discussed and analysed in section 4 .

\section{Derivation of the artificial pressure equations}

Pseudo-compressibility methods are based on simplifications of the Navier-Stokes equations in the limit of low Mach number. This section briefly presents a derivation of the main approaches relevant to this study, with the aim to highlight the differences between the various methods. Two main types of approximation are used to derive the artificial pressure equations investigated in the paper. First, the Mach number is artifically increased to reduce the number of timesteps required for the numerical resolution of the flow. Second, the governing equations are simplified in order to ensure a constant density and thermodynamical pressure and to reduce the number of operations per timestep. To perform the first step, three flows will be defined in the following. The flow $F$ is an arbitrary flow to numerically resolve. In the flow $F^{\prime}$, the velocity of the fluid is increased compared to the 
flow $F$ in order to increase the Mach number. Using a change of variable, the artificial flow $F^{\prime \prime}$ may be defined, with the same typical fluid velocity as the flow $F$, but instead an artificially reduced speed of sound. The crucial point is that the flow $F^{\prime \prime}$ is associated with the same boundary conditions as the flow $F$ but a modified system of equations. The simplification of this system of equations leads to the simplified forms investigated in the paper.

Consider the flow $F$ of an ideal gas, associated with the spatiotemporal fields $(U, \rho, P)$ and characterised by a length scale $x^{b}$, a velocity scale $U^{b}$, a time scale $t^{b}=x^{b} / U^{b}$, a temperature scale $T^{b}$, a density scale $\rho^{b}$ and a pressure scale $P^{b}=r \rho^{b} T^{b}$. The Mach number $M a$ representative of the flow is $M a=U^{b} / c^{b}$, where $c^{b}=\sqrt{\gamma r T^{b}}$ is the representative speed of sound, with $\gamma=C_{p} / C_{v}$ the adiabatic index and $r$ is the ideal gas specific constant. Suppose that this flow can be modelled by the compressible Navier-Stokes equations, in which dissipation has been neglected in the pressure evolution equation:

$$
\begin{gathered}
\frac{\partial \rho}{\partial t}+\frac{\partial \rho U_{j}}{\partial x_{j}}=0 \\
\frac{\partial \rho U_{i}}{\partial t}+\frac{\partial \rho U_{j} U_{i}}{\partial x_{j}}=-\frac{\partial P}{\partial x_{i}}+\frac{\partial}{\partial x_{j}}\left(\mu\left(\frac{\partial U_{i}}{\partial x_{j}}+\frac{\partial U_{j}}{\partial x_{i}}\right)\right)-\frac{2}{3} \frac{\partial}{\partial x_{i}}\left(\mu \frac{\partial U_{j}}{\partial x_{j}}\right), \\
\frac{\partial P}{\partial t}+\frac{\partial U_{j} P}{\partial x_{j}}=(\gamma-1) \frac{\partial}{\partial x_{j}}\left(\lambda \frac{\partial T}{\partial x_{j}}\right)+P(1-\gamma) \frac{\partial U_{j}}{\partial x_{j}} \\
P=r \rho T
\end{gathered}
$$

with some initial and boundary conditions which are functions of $\left(x^{b}, U^{b}, T^{b}, \rho^{b}\right)$. Without loss of generality, we included no body forces and no heat sources. If the Mach number $M a$ is small, the numerical resolution of these equations with an explicit time stepping is inefficient because the speed of sound is large compared to the velocity of the fluid.

Let $\alpha>1$ be a constant. Provided that $\alpha M a$ remains small, the flow $F$ can be approximated by another flow $F^{\prime}$, associated with $\left(U^{\prime}, \rho^{\prime}, P^{\prime}\right)$, with the same geometry, temperature scale $T^{b}$, Prandtl and Reynolds numbers but characterised by the Mach number $M a^{\prime}=\alpha M a$. The flow $F^{\prime}$ is more efficient to resolve than the $F$ because the disparity between the velocity of the fluid and the velocity of acoustic waves is reduced. This is the essence of weakly compressible (WC) methods, in which the compressible Navier-Stokes equations are used to simulate incompressible or almost compressible flows [23, 27, 7]. However, the flow $F^{\prime}$ may only approximate the flow $F$ in the nondimensionalised sense, since it necessarily has a different time scale $t^{\prime b}=t^{b} / \alpha$, velocity scale $U^{\prime b}=\alpha U^{b}$, density scale $\rho^{b}=\rho^{b} / \alpha$ and pressure scale $P^{\prime b}=P^{b} / \alpha$ and thus requires adjusted initial and boundary conditions. An asymptotic development of each nondimensionalised variable as a function of the squared Mach number [40, 49, 43] suggests the approximations $U / U^{b} \approx v_{0}$, $\rho / \rho^{b} \approx \varrho_{0}$ and $P / P^{b} \approx \pi_{0}+M a^{2} \pi_{1}$, where $v_{0}, \varrho_{0}, \pi_{0}$ and $\pi_{1}$ do not depend on the Mach number and $\pi_{0}$ is constant in space. Thus, $U^{\prime} \approx \alpha U, \rho^{\prime} \approx \rho / \alpha, P_{0}^{\prime} \approx P_{0} / \alpha$ and $P_{1}^{\prime} \approx \alpha P_{1}$, with $P_{0}=P^{b} \pi_{0}$ and $P_{1}=P^{b} M a^{2} \pi_{1}$.

We will use a change of variables to recover the time, velocity, density and pressure scales of $F$ and instead modify the system of equations. We let $t^{\prime \prime}=\alpha t^{\prime}, U^{\prime \prime}=U^{\prime} / \alpha, \rho^{\prime \prime}=$ $\alpha \rho^{\prime}, P_{0}^{\prime \prime}=\alpha P_{0}^{\prime}$ and $P_{1}^{\prime \prime}=P_{1}^{\prime} / \alpha$, such that $U^{\prime \prime} \approx U, \rho^{\prime \prime} \approx \rho, P_{0}^{\prime \prime} \approx P_{0}$ and $P_{1}^{\prime \prime} \approx P_{1}$. The resolution of the flow $F^{\prime}$ with the compressible Navier-Stokes equations (4-7) is equivalent to the resolution of the artificial flow $F^{\prime \prime}$, solution of the modified system of equations:

$$
\frac{\partial \rho^{\prime \prime}}{\partial t^{\prime \prime}}+\frac{\partial \rho^{\prime \prime} U_{j}^{\prime \prime}}{\partial x_{j}}=0
$$


Analysis of artificial pressure equations in numerical simulations of a turbulent channel flow

$$
\begin{gathered}
\frac{\partial \rho^{\prime \prime} U_{i}^{\prime \prime}}{\partial t^{\prime \prime}}+\frac{\partial \rho^{\prime \prime} U_{j}^{\prime \prime} U_{i}^{\prime \prime}}{\partial x_{j}}=-\frac{\partial P_{1}^{\prime \prime}}{\partial x_{i}}+\frac{\partial}{\partial x_{j}}\left(\mu\left(\frac{\partial U_{i}^{\prime \prime}}{\partial x_{j}}+\frac{\partial U_{j}^{\prime \prime}}{\partial x_{i}}\right)\right)-\frac{2}{3} \frac{\partial}{\partial x_{i}}\left(\mu \frac{\partial U_{j}^{\prime \prime}}{\partial x_{j}}\right), \\
\frac{\partial P_{1}^{\prime \prime}}{\partial t^{\prime \prime}}+\frac{\partial U_{j}^{\prime \prime} P_{1}^{\prime \prime}}{\partial x_{j}}=\frac{(\gamma-1)}{\alpha^{2}} \lambda \frac{\partial T}{\partial x_{j}}+\left(P_{1}^{\prime \prime}(1-\gamma)-\frac{\gamma P_{0}^{\prime \prime}}{\alpha^{2}}\right) \frac{\partial U_{j}^{\prime \prime}}{\partial x_{j}}-\frac{1}{\alpha^{2}} \frac{\partial P_{0}^{\prime \prime}}{\partial t}, \\
P_{0}^{\prime \prime}+\alpha^{2} P_{1}^{\prime \prime}=r \rho^{\prime \prime} T,
\end{gathered}
$$

with the same time, velocity, density and pressure scales (and thus initial and boundary conditions) as the flow $F$. The decomposition of pressure in (8-11) is necessary to properly scale the pressure variations. The naive scaling $P^{\prime \prime}=\alpha P$ leads to a system of equations akin to the pressure gradient scaling (PGS) method, in which pressure variations are amplified by a factor $\alpha^{2}[55]$. The artificial flow $F^{\prime \prime}$ approximates the flow $F$ in both the dimensional and nondimensionalised sense and is as efficient to resolve as the flow $F^{\prime}$. The flow $F^{\prime}$ has the same speed of sound as the flow $F$ but a larger fluid velocity. The flow $F^{\prime \prime}$ has the same typical fluid velocity as the flow $F$ but an artificially low speed of sound.

Several approximations may be applied on the system (8-11) to reduce the number of independent variables and the numerical cost of its resolution and with the intent to bring its solution closer to the incompressible solution. In order to impose a constant density without adding a constraint on the divergence of velocity, the continuity equation (8) is removed from the system. In accordance, the time derivative of $P_{0}^{\prime \prime}$ is neglected in equation (10) Besides, we can neglect $P_{1}^{\prime \prime}$ as compared to $P_{0}^{\prime \prime} / \alpha^{2}$ in equation (10) as the thermodynamical pressure $P_{0}$ is typically very large compared to the mechanical pressure $P_{1}$ in low Mach number flows. Finally, the viscous term of the momentum conservation equation (9) may be simplified by neglecting the contribution of the velocity divergence. This leads to:

$$
\begin{gathered}
\frac{\partial \rho^{\prime \prime} U_{i}^{\prime \prime}}{\partial t^{\prime \prime}}+\frac{\partial \rho^{\prime \prime} U_{j}^{\prime \prime} U_{i}^{\prime \prime}}{\partial x_{j}}=-\frac{\partial P_{1}^{\prime \prime}}{\partial x_{i}}+\frac{\partial}{\partial x_{j}}\left(\mu \frac{\partial U_{i}^{\prime \prime}}{\partial x_{j}}\right), \\
\frac{\partial P_{1}^{\prime \prime}}{\partial t^{\prime \prime}}+\frac{\partial U_{j}^{\prime \prime} P_{1}^{\prime \prime}}{\partial x_{j}}=\frac{(\gamma-1)}{\alpha^{2}} \frac{\partial}{\partial x_{j}}\left(\lambda \frac{\partial T}{\partial x_{j}}\right)-\frac{\gamma P_{0}^{\prime \prime}}{\alpha^{2}} \frac{\partial U_{j}^{\prime \prime}}{\partial x_{j}}, \\
P_{0}^{\prime \prime}+\alpha^{2} P_{1}^{\prime \prime}=r \rho^{\prime \prime} T .
\end{gathered}
$$

Injecting 14 into 13 and neglecting the variations of the fluid properties with pressure, we obtain

$$
\frac{\partial P_{1}^{\prime \prime}}{\partial t^{\prime \prime}}+\frac{\partial U_{j}^{\prime \prime} P_{1}^{\prime \prime}}{\partial x_{j}}=\frac{\gamma \nu}{\operatorname{Pr}} \frac{\partial^{2} P_{1}^{\prime \prime}}{\partial x_{j}^{2}}-\frac{\gamma P_{0}^{\prime \prime}}{\alpha^{2}} \frac{\partial U_{j}^{\prime \prime}}{\partial x_{j}}
$$

with $\operatorname{Pr}=\gamma \mu r /(\lambda(\gamma-1))$ the Prandtl number. Following Clausen [15], we use $\operatorname{Pr}=\gamma$ as a Prandtl number and define the artificial speed of sound $c=\sqrt{\gamma P_{0}^{\prime \prime} /\left(\rho \alpha^{2}\right)} \approx c^{b} / \alpha$. This leads to the artificial compressibility method with a convective and a diffusive term, given by equations (1) and

$$
\frac{\partial P}{\partial t}=-\frac{\partial U_{j} P}{\partial x_{j}}-\rho c^{2} \frac{\partial U_{j}}{\partial x_{j}}+\nu \frac{\partial^{2} P}{\partial x_{j}^{2}}
$$

This equation is equivalent to the GP equation [60] and the pressure equation of the EDAC method [15]. Neglecting the convective term this equation leads to the artificial compressibility method with a diffusive term, given by equations (1) and

$$
\frac{\partial P}{\partial t}=-\rho c^{2} \frac{\partial U_{j}}{\partial x_{j}}+\nu \frac{\partial^{2} P}{\partial x_{j}^{2}}
$$


while neglecting the diffusive term leads to the artificial compressibility method with a convective term, given by equations (1) and

$$
\frac{\partial P}{\partial t}=-\frac{\partial U_{j} P}{\partial x_{j}}-\rho c^{2} \frac{\partial U_{j}}{\partial x_{j}} .
$$

Finally, neglecting all but the divergence term in the GP equation leads to the artificial compressibility equation (3) without subiteration. In the following, we will compare numerical methods based on equations (16), (17), (18) and (3) in order to assess the relevance of each approximation. A compressible solver based on equations (4-7) will also be used to study weakly compressible methods.

\section{Numerical study configuration}

We consider a fully developed three-dimensional turbulent channel flow. We denote $x$ the streamwise direction, $y$ the wall-normal direction and $z$ the spanwise direction. The geometry is periodic in the $x$ and $z$ directions. The domain size is $4 \pi h \times 2 h \times 2 \pi h$. The flow is isothermal and incompressible. The mass flow rate is imposed at value corresponding, in a direct numerical simulation, to a friction Reynolds number $R e_{\tau}=180$, where $R e_{\tau}$ is defined as $R e_{\tau}=U_{\tau} h / \nu$ with $U_{\tau}=\sqrt{\nu\left(\partial_{y}\left\langle U_{x}\right\rangle\right)_{\omega}}$ the friction velocity, where the subscript $\omega$ denotes wall values.

We carry out coarse simulations of the channel on a coarse mesh, and direct numerical simulations of the channel on a fine mesh. The two meshes are uniform in the homogeneous directions and follow in the wall-normal direction a hyperbolic tangent law of the form

$$
y_{k}=h\left(1+\frac{1}{a} \tanh \left[\left(\frac{k-1}{N_{y}-1}-1\right) \tanh ^{-1}(a)\right]\right),
$$

where $a$ is the mesh dilatation parameter and $N_{y}$ the number of grid points. The coarse mesh contains $48 \times 50 \times 48$ cells and the cell sizes in wall-units are $\Delta_{x}^{+}=68, \Delta_{y, \omega}^{+}=0.50$ at the wall and $\Delta_{y, c}^{+}=25$ at the center and $\Delta_{z}^{+}=34$. The fine mesh contains $384 \times 266 \times 384$ cells and the cell sizes in wall-units are $\Delta_{x}^{+}=5.8, \Delta_{y, \omega}^{+}=0.0 .085$ at the wall and $\Delta_{y, c}=2.9$ at the center and $\Delta_{z}^{+}=2.9$. The same mass flow rate is imposed with the coarse and fine meshes but accordingly a different wall shear stress may be obtained, as seen in Dupuy et al. $[19,20]$.

We use a finite difference method written in a divergence form in a staggered grid system [46, 51]. The setting is illustrated in Figure 1 in the two-dimensional case. A fourth-order centred scheme is used for momentum convection and a second-order centred scheme is used for momentum diffusion [17]. We use the following discretisations for the terms of the pressure evolution equation:

$$
\begin{aligned}
(\nabla \cdot U)_{i j k}= & \frac{u_{i+1 j k}-u_{i j k}}{\Delta x_{i}}+\frac{v_{i j+1 k}-v_{i j k}}{\Delta y_{j}}+\frac{w_{i j k+1}-w_{i j k}}{\Delta z_{k}}, \\
(\nabla \cdot(U P))_{i j k}= & \frac{u_{i+1 j k}\left(P_{i+1 j k}+P_{i j k}\right)-u_{i j k}\left(P_{i j k}+P_{i-1 j k}\right)}{2 \Delta x_{i}} \\
& +\frac{v_{i j+1 k}\left(P_{i j+1 k}+P_{i j k}\right)-v_{i j k}\left(P_{i j k}+P_{i j-1 k}\right)}{2 \Delta y_{j}} \\
& +\frac{w_{i j k+1}\left(P_{i j k+1}+P_{i j k}\right)-w_{i j k}\left(P_{i j k}+P_{i j k-1}\right)}{2 \Delta z_{k}},
\end{aligned}
$$




$$
\begin{aligned}
(\Delta P)_{i j k}= & \frac{\left(P_{i+1 j k}-P_{i j k}\right) /\left(\Delta x_{i+1}+\Delta x_{i}\right)-\left(P_{i j k}-P_{i-1 j k}\right) /\left(\Delta x_{i}+\Delta x_{i-1}\right)}{\Delta x_{i} / 2} \\
& +\frac{\left(P_{i j+1 k}-P_{i j k}\right) /\left(\Delta y_{j+1}+\Delta y_{j}\right)-\left(P_{i j k}-P_{i j-1 k}\right) /\left(\Delta y_{j}+\Delta y_{j-1}\right)}{\Delta y_{j} / 2} \\
& +\frac{\left(P_{i j k+1}-P_{i j k}\right) /\left(\Delta z_{k+1}+\Delta z_{k}\right)-\left(P_{i j k}-P_{i j k-1}\right) /\left(\Delta z_{k}+\Delta z_{k-1}\right)}{\Delta z_{k} / 2} .
\end{aligned}
$$

The time scheme is given by a semi-implicit third-order Runge-Kutta method. For the system of equations put in the form $\partial_{t} P=G(\boldsymbol{U}, P)$ and $\partial_{t} \boldsymbol{U}=F(\boldsymbol{U}, P)$, the algorithm can be expressed as [64]:

$$
\begin{aligned}
P^{n+1 / 3} & =P^{n}+b_{1} \Delta_{t} \ell_{1}, & \boldsymbol{U}^{n+1 / 3} & =\boldsymbol{U}^{n}+b_{1} \Delta_{t} k_{1}, \\
P^{n+3 / 4} & =P^{n}+b_{2} \Delta_{t} \ell_{2}, & \boldsymbol{U}^{n+3 / 4} & =\boldsymbol{U}^{n}+b_{2} \Delta_{t} k_{2}, \\
P^{n+1} & =P^{n}+b_{3} \Delta_{t} \ell_{3}, & \boldsymbol{U}^{n+1} & =\boldsymbol{U}^{n}+b_{3} \Delta_{t} k_{3},
\end{aligned}
$$

using the estimated slopes

$$
\begin{array}{ll}
\ell_{1}=G\left(\boldsymbol{U}^{n}, P^{n}\right), & k_{1}=F\left(\boldsymbol{U}^{n}, P^{n+1 / 3}\right), \\
\ell_{2}=G\left(\boldsymbol{U}^{n+1 / 3}, P^{n+1 / 3}\right)+a_{2} \ell_{1}, & k_{2}=F\left(\boldsymbol{U}^{n+1 / 3}, P^{n+3 / 4}\right)+a_{2} k_{1}, \\
\ell_{3}=G\left(\boldsymbol{U}^{n+3 / 4}, P^{n+3 / 4}\right)+a_{3} \ell_{2}, & k_{3}=F\left(\boldsymbol{U}^{n+3 / 4}, P^{n+1}\right)+a_{3} k_{2},
\end{array}
$$

with the constants $a_{2}=-5 / 9, a_{3}=-153 / 128, b_{1}=1 / 3, b_{2}=15 / 16$ and $b_{3}=8 / 15$. We use the pressure at the next intermediate timestep to compute the increment of velocity in equations 26, 27 and 28 to improve the stability of the numerical method. Since the numerical schemes are explicit in time and local in space, the numerical method does not require the resolution of a system of coupled algebraic equations and is well-suited to parallel implementation. The timestep is set according to the following Courant-Friedrichs-Lewy condition:

$$
\Delta_{t}^{+}=\frac{M a_{\tau}}{R e_{\tau}} \Delta_{y, \omega}^{+}
$$

where $\Delta_{t}^{+}=\Delta_{t} U_{\tau} / h$ is the timestep in wall units and $M a_{\tau}=U_{\tau} / c$ is the artificial friction Mach number. Since the timestep is smaller than in the corresponding simulation with a projection method, it is generally small compared to the flow physics and there are no accuracy issues associated with the timestep. With the fine mesh, the simulations are in addition mesh converged [17] and thus do not depend on the exact numerical method, provided that it is stable. A no-slip boundary condition is used at the walls. If the pressure equation does not involve a diffusive term, no wall boundary condition is required for the pressure. If the pressure equation involves a diffusive term, the pressure gradient at the wall is imposed to $\partial_{y} P=\mu \partial_{y}^{2} U_{y}$ using a four-point Lagrange polynomial interpolation of the velocity to compute the second-order derivative, following [29], and the density convection is discretised using the third-order QUICK (quadratic upstream interpolation for convective kinetics) scheme [38]. This is performed using the TrioCFD software [10].

We performed simulations of the channel using artificial pressure equations. The artificial Mach numbers investigated on the coarse and fine meshes are reported in Table 1. We used the volumetric flow rate in order to define the Mach number $M a$. To analyse the results, we also performed simulations of the channel using a projection method. The simulations with a projection method have been carried out using the same mesh, the same domain size and the same numerical schemes as the simulations using an artificial pressure equation. The numerical set-up has been validated in previous papers $[17,18]$ against the reference data of Moser et al. [47], Bolotnov et al. [8], Vreman and Kuerten [62] and Lee and Moser [37]. 


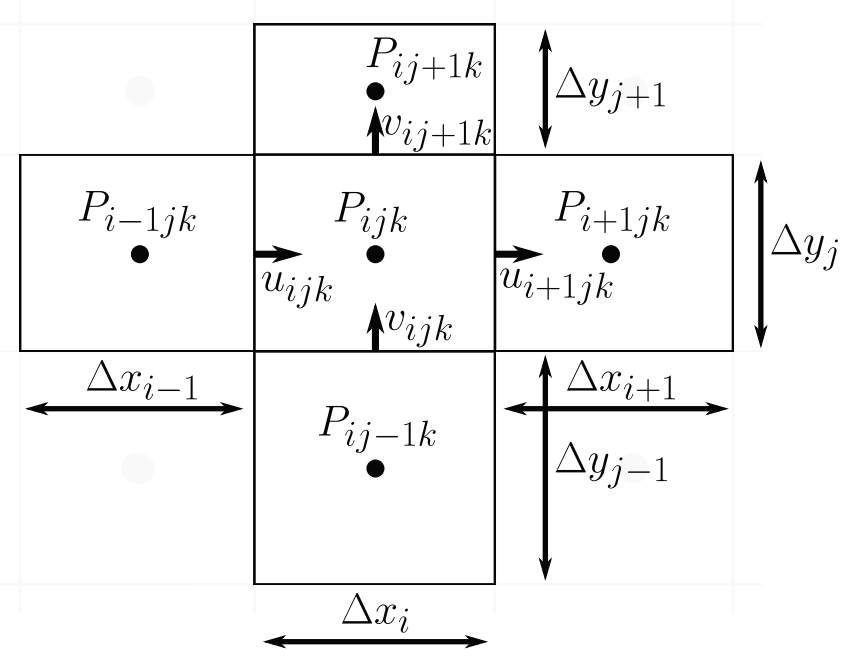

Figure 1 - Staggered grid system.

\begin{tabular}{lcc} 
Mesh & $M a$ & $M a_{\tau}$ \\
\hline Coarse & 0.78 & 0.050 \\
Coarse & 0.55 & 0.035 \\
Coarse & 0.39 & 0.025 \\
Coarse & 0.25 & 0.016 \\
Coarse & 0.18 & 0.011 \\
Coarse & 0.12 & 0.008 \\
Coarse & 0.08 & 0.005 \\
Fine & 1.00 & 0.064 \\
Fine & 0.50 & 0.032 \\
Fine & 0.25 & 0.016 \\
Fine & 0.10 & 0.006
\end{tabular}

Table 1 - Artificial Mach numbers (associated with the volumetric flow rate) and artificial friction Mach numbers of the simulations on the coarse and fine meshes.

\section{Results and discussion}

\subsection{Artificial compressibility method}

We first study the numerical simulations of the turbulent channel flow with the artificial compressibility method, given by equations (1) and (3). The simulations are stable for all artificial Mach number investigated (see Table 1) with our meshes, timestep and numerical method. The velocity divergence is smooth and exhibits a clear correlation with the pressure field (Figure 2(a)). The first-order and second-order statistics of turbulence given by the fine simulations are reported in Figure 3. Our numerical results support the fact that as the artificial Mach number tends towards zero, the simulations converge to the incompressible solution, here represented by the corresponding simulation with a projection method. At $M a=1.00$, the friction velocity is underestimated and the standard deviation of streamwise velocity overestimated, while the standard deviation of the other velocity components and of pressure is underestimated. At $M a=0.10$, a very good gen- 
eral agreement is obtained. A slight difference of the results with the reference projection simulation is visible for the standard deviation of streamwise and wall-normal velocity and of pressure, while the mean streamwise velocity, the covariance of streamwise and wallnormal velocity, the standard deviation of streamwise velocity and the mean pressure are almost identical. On the coarse mesh (Figure 4), the simulations also converge towards the incompressible solution, which differs from the results of the direct numerical simulation because of the insufficient mesh resolution. At $M a=0.08$, the results of the artificial compressibility simulations and the reference projection simulation are indistinguishable.

The rate of convergence of some turbulence statistics is represented in Figure 5. A quadratic $\mathcal{O}\left(M a^{2}\right)$ rate of convergence is expected for the artificial compressibility method [14, $34,52,53]$. Numerically, the quadratic convergence rate of the second-order turbulence statistics is very clear for the coarse simulations at high artificial Mach number (Figure 5). At low artificial Mach number, our results deviate from this expected behaviour. The quadratic convergence rate can be used to extrapolate the results of two or more simulations to an artificial Mach number of zero, as suggested by Ohwada and Asinari [52]. We consider for the sake of simplicity the statistics of two simulations $s_{1}$ and $s_{2}$ at the respective artificial Mach numbers $M a_{1}$ and $M a_{2}$. The results are extrapolated to $M a_{0}=0$ using $s_{0}(y)=\left(s_{1}(y)^{2} / M a_{1}^{2}-s_{2}(y)^{2} / M a_{2}^{2}\right) /\left(1 / M a_{1}^{2}-1 / M a_{2}^{2}\right)$ for each wall-normal coordinate $y$. Notice that no interpolation is required since the two simulations are carried out on the same mesh. The extrapolation of the simulations on the coarse mesh at $M a=0.25$ and $M a=0.39$ is more accurate than the simulation at $M a=0.12$ (Figure 6). However, it requires $20 \%$ less computational time since the timestep of the simulation is inversely proportional to the artificial Mach number. Similarly, the extrapolation of the simulations at $M a=0.39$ and $M a=0.55$ more accurate than the simulation at $M a=0.25$ but requires $10 \%$ less computational time. This procedure thus accelerates the convergence of the artificial compressibility method. Given these promising results, it would be interesting to examine the applicability of the extrapolation method in more complex fluid flows. 

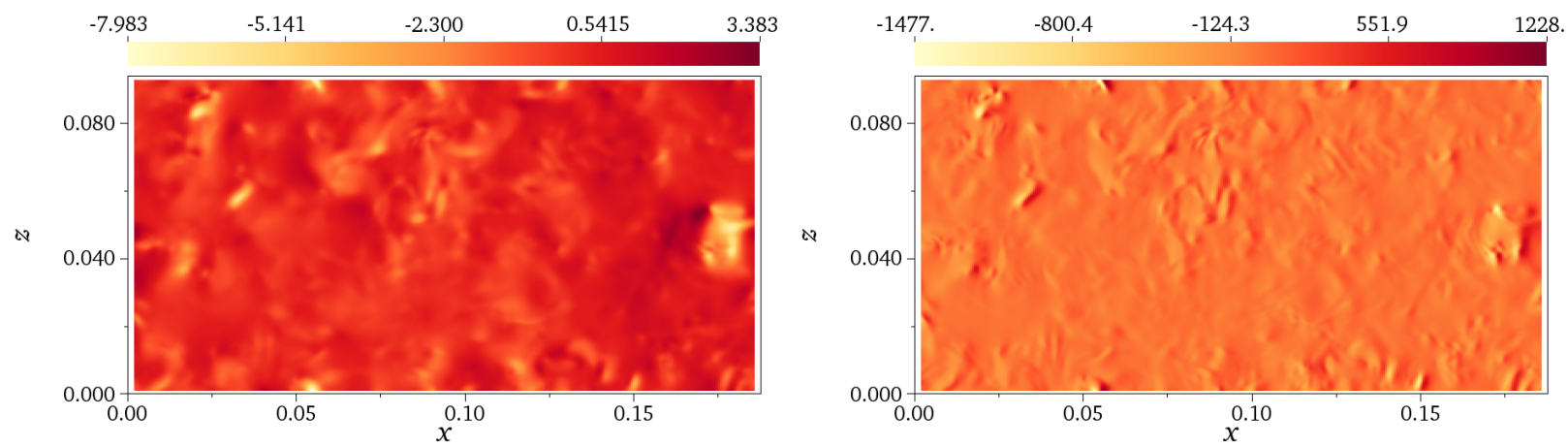

(a) Artificial compressibility method
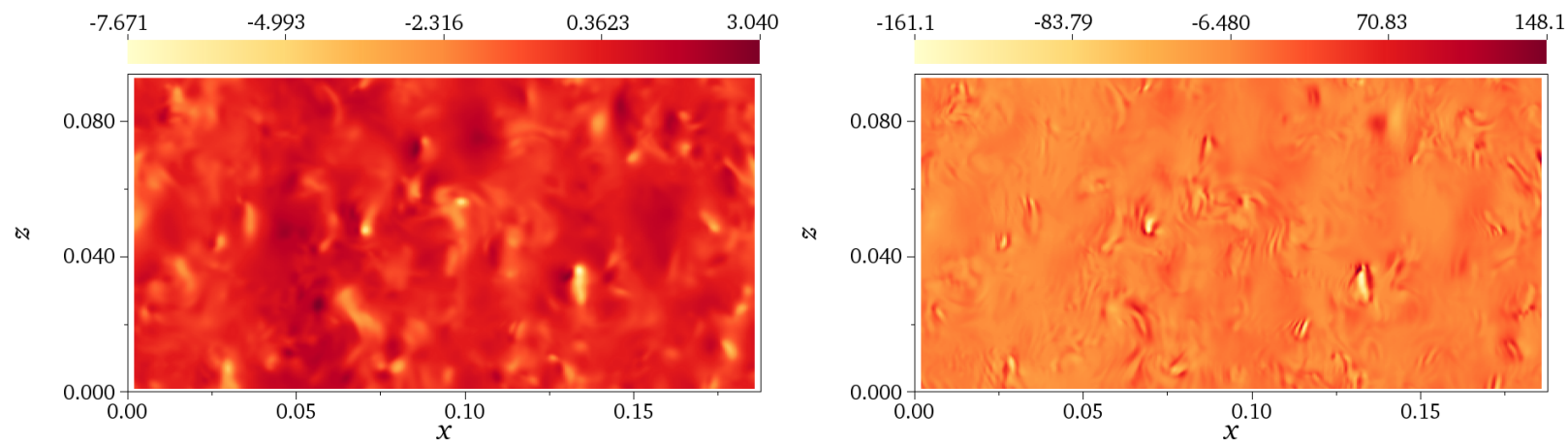

(b) Artificial compressibility method with a convective term

Figure 2 - Fields of instantaneous pressure (left) and velocity divergence (right) in wall units at the center of the channel ( $y=h$ plane) for simulations on the fine mesh at $M a=0.25$ using the artificial compressibility method (3) and the artificial compressibility method with a convective term (18). The velocity divergence is premultiplied by $10^{6}$. 

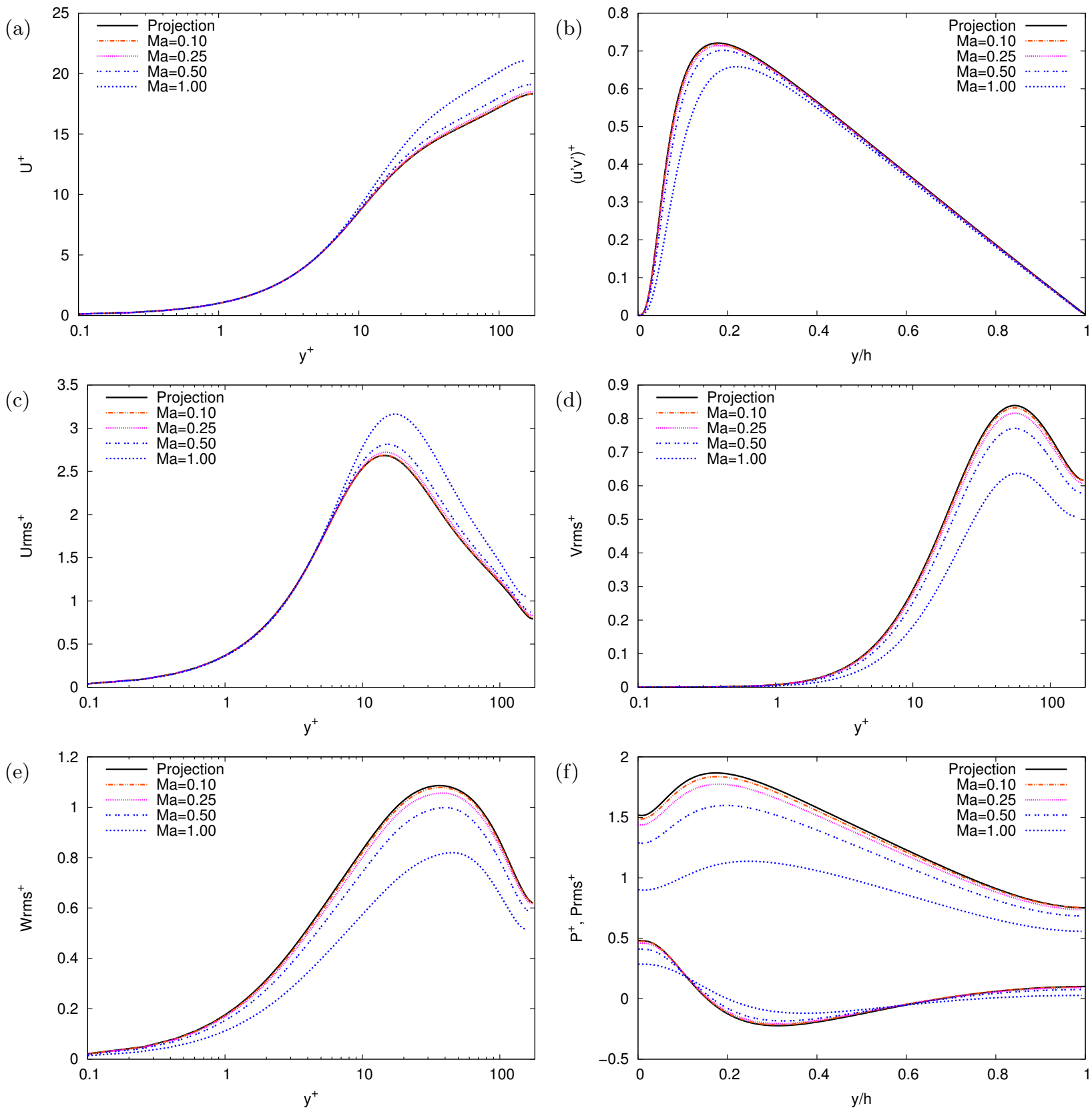

Figure 3 - Turbulence statistics for artificial compressibility simulations on the fine mesh and the corresponding simulation with a projection method: (a) mean streamwise velocity $\left\langle U_{x}\right\rangle$, (b) covariance of streamwise and wall-normal velocity $\left\langle u_{x}^{\prime} u_{y}^{\prime}\right\rangle$, (c) standard deviation of streamwise velocity $\sqrt{\left\langle u_{x}^{\prime 2}\right\rangle}$, (d) standard deviation of wall-normal velocity $\sqrt{\left\langle u_{y}^{\prime 2}\right\rangle}$, (e) standard deviation of spanwise velocity $\sqrt{\left\langle u_{z}^{\prime 2}\right\rangle}$, (f, bottom) mean pressure $\langle P\rangle$, (f, top) standard deviation of pressure $\sqrt{\left\langle p^{\prime 2}\right\rangle}$. 

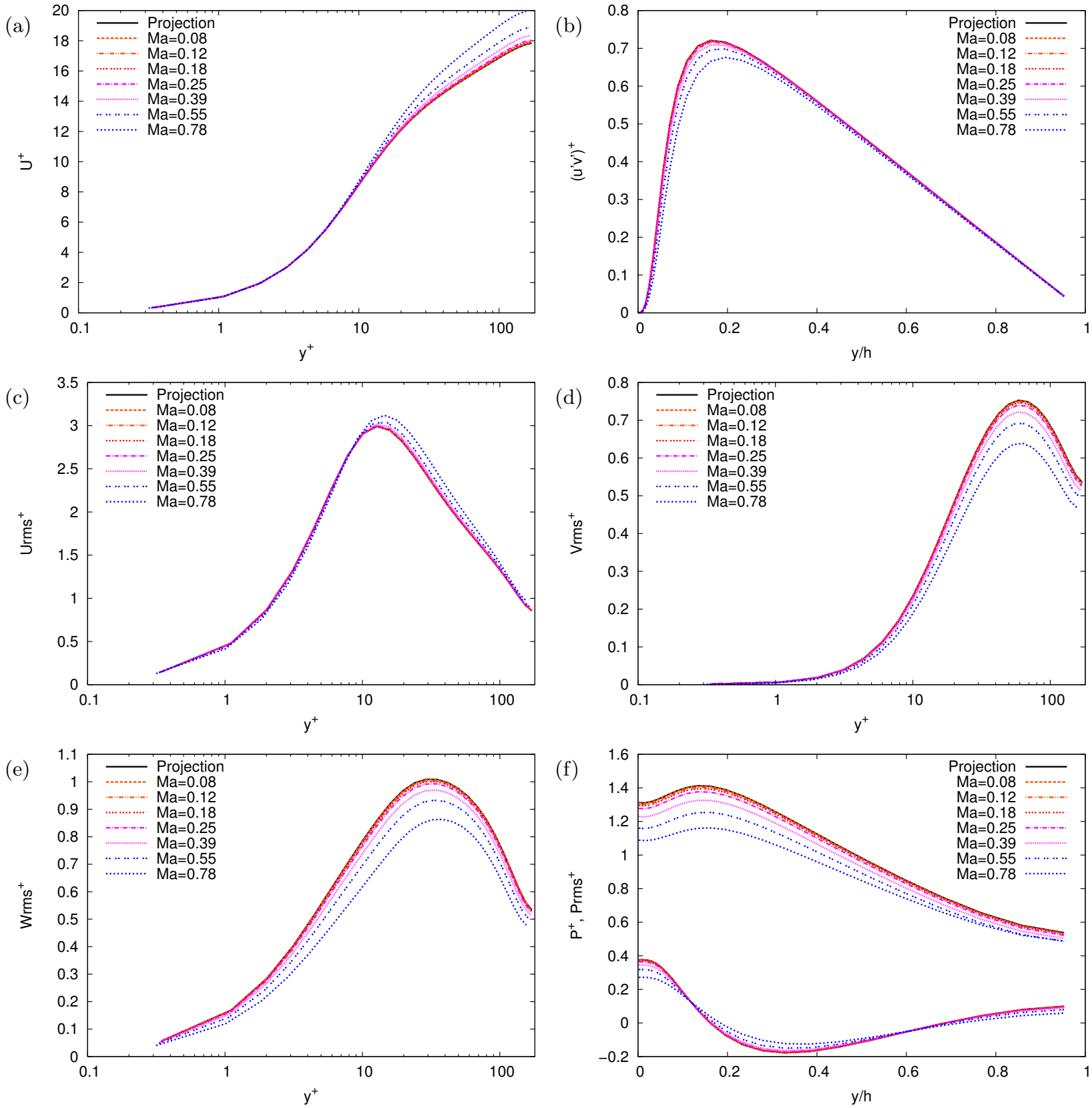

Figure 4 - Turbulence statistics for artificial compressibility simulations on the coarse mesh and the corresponding simulation with a projection method. See Figure 3 for labels. 
Analysis of artificial pressure equations in numerical simulations of a turbulent channel flow
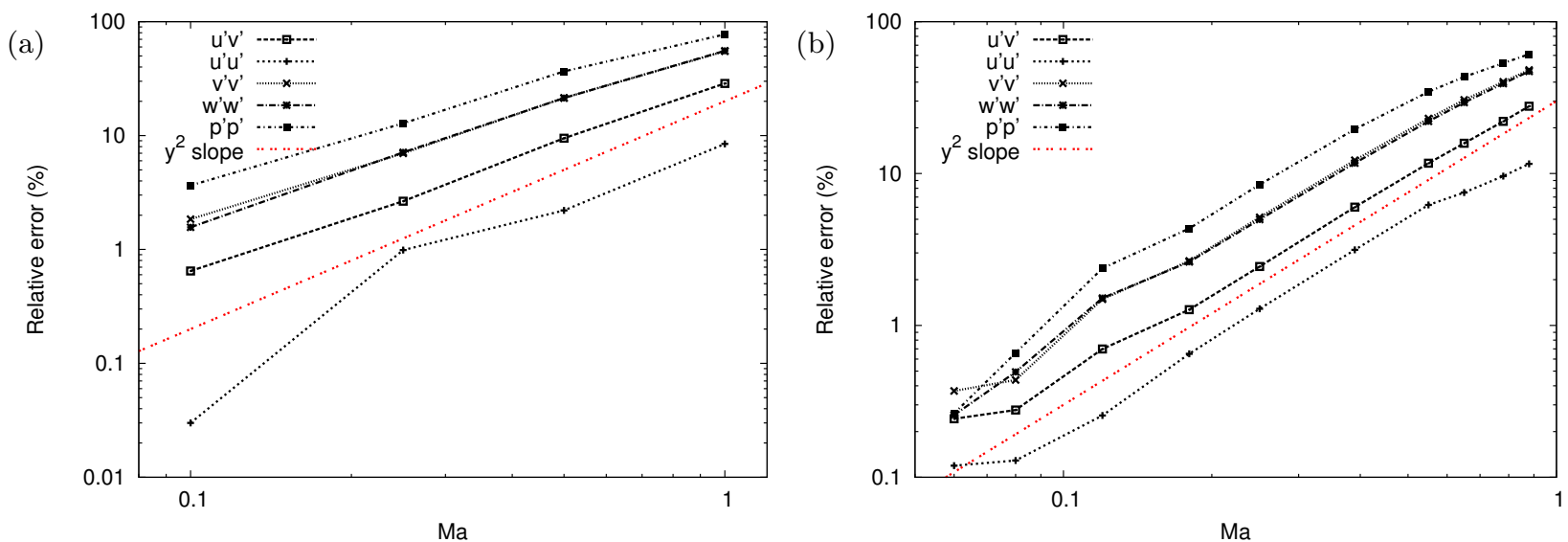

Figure 5 - Relative error between the artificial compressibility simulations on the fine mesh (a) or the coarse mesh (b) and the corresponding simulation with a projection method for the maximum value of the covariance of streamwise and wall-normal velocity $\left\langle u_{x}^{\prime} u_{y}^{\prime}\right\rangle$, the variance of streamwise velocity $\left\langle u_{x}^{\prime 2}\right\rangle$, wall-normal velocity $\left\langle u_{y}^{\prime 2}\right\rangle$, spanwise velocity $\left\langle u_{z}^{\prime 2}\right\rangle$, and pressure $\left\langle p^{2}\right\rangle$. 

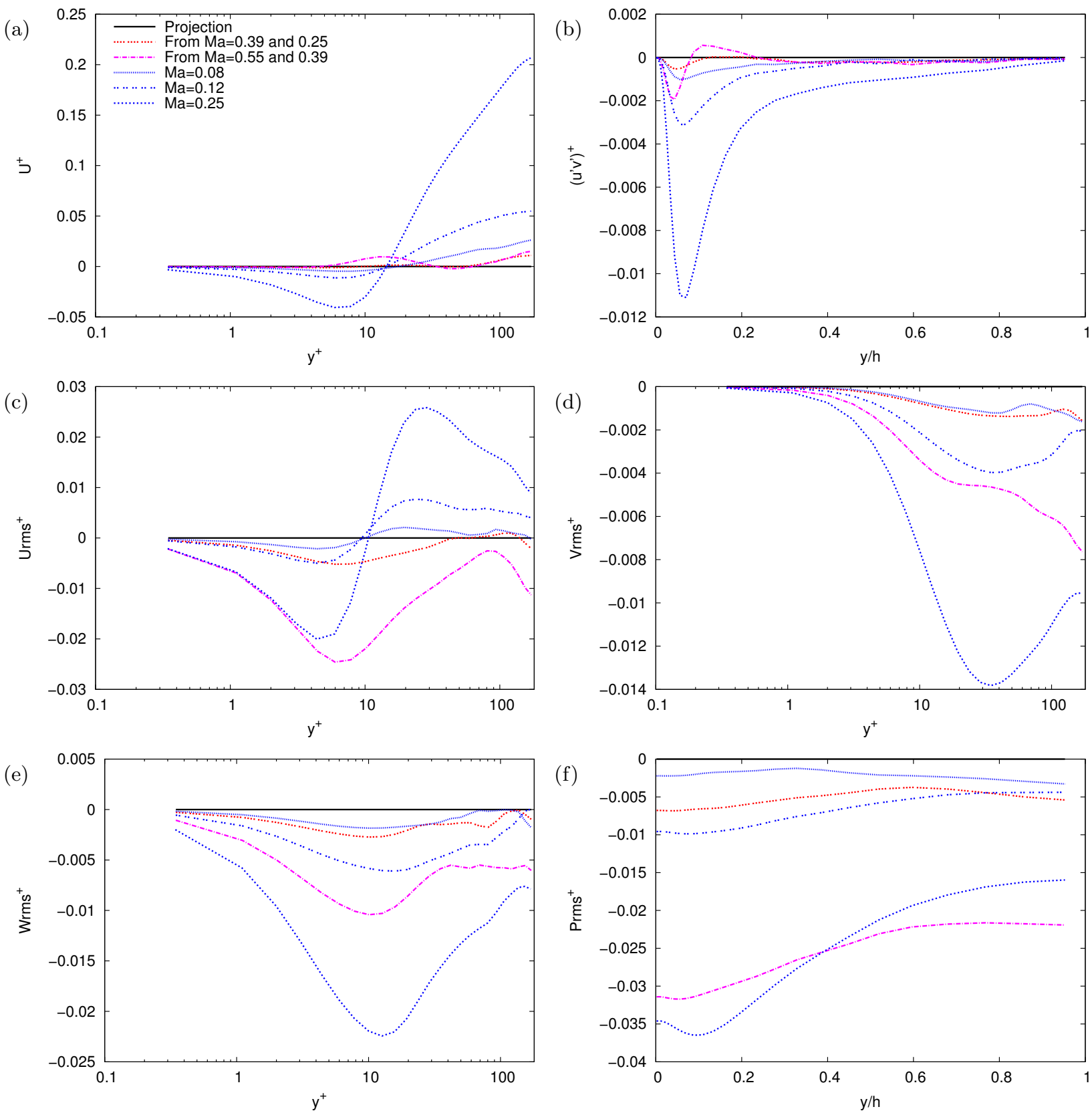

Figure 6 - Turbulence statistics for extrapolated artificial compressibility simulations on the coarse mesh and artificial compressibility simulations without extrapolation, presented as differences from the reference projection simulation. See Figure 3 for labels. 


\subsection{Comparison of artificial pressure equations}

As discussed in section 2, several numerical methods can be identified as intermediate approximations between the compressible Navier-Stokes equations and the artificial compressibility method. We study the artificial compressibility method with a convective and a diffusive term (16), the artificial compressibility method with a diffusive term (17) and the artificial compressibility method with a convective term(18). We compare the standard deviation of velocity divergence obtained with the three methods on the coarse mesh at $M a=0.12$ in Figure 7. The addition of a diffusive term to the pressure evolution equation has a negligible impact on the fluctuations of the velocity divergence. On the other hand, the addition of a convective term reduces the standard deviation of velocity divergence away from the wall. This has a large impact on the accuracy of the simulation.

Figure 8 shows that with a convective term, simulations on the coarse mesh are in a good general agreement with the reference projection simulation at $M a=0.55$. In particular, the profiles of the Reynolds stresses are superimposed below $M a=0.39$ and remain within a moderate error range at $M a=0.78$. The standard deviation of pressure is also improved for most artificial Mach number but does not converge exactly to the reference projection simulation at low artificial Mach numbers. These results are surprising since the convective term could be expected to be negligible given the disparity between the convective velocity and the speed of sound [9, 15,60], but support the analysis of Kreiss et al. [34], which first considered the artificial compressibility equations with a convective term. Besides, the convective term is required to ensure the Galilean invariance of the pressure evolution equation. The main phenomenon affecting pressure is the transport by the velocity of the fluid, which is captured by the convective term, allowing higher artificial Mach numbers. We expect this behaviour to only be valid for unsteady flows as equation (18) does not ensure the divergence-free condition in steady solenoidal flows while this condition is ensured using the original artificial compressibility equation (3). The simulation on a fine mesh at $M a=0.25$, given in Figure 9, confirms the powerful influence of the convective term for direct numerical simulations. The velocity divergence remains clearly correlated with the pressure field (Figure 2(b)), but its magnitude is reduced by a factor of 10 at the center of the channel.

The addition of a diffusive term to the pressure evolution equation has a negligible impact on the prediction of the Reynolds stresses (Figure 10). However, it improves the convergence of the standard deviation of pressure. Below $M a=0.25$, the artificial compressibility method with a convective and a diffusive term gives identical results to the reference projection simulation for all first-order and second-order statistics of turbulence. This represents a three-fold speedup compared to the artificial compressibility method. Higher-order statistics, such as the skewness and flatness factors of velocity are also well represented (Figure 11).

The artificial compressibility method with a convective and a diffusive term is compared to the resolution of the compressible Navier-Stokes equations in Figure 12. For a given Mach number, the results of the compressible solver deviate more significantly from the reference projection simulation than the artificial compressibility method. This can be explained by the fact that the density is imposed in the artificial compressibility method while the continuity equation is resolved in the compressible solver. This point also makes the artificial compressibility method simpler and slightly reduces the memory requirements and the computational time per timestep. Note also that the modification of the Mach number requires the adjustment of the initial and boundary conditions in the compressible 
solver while it is included in the numerical method in artificial compressibility methods (see section 2). The computational efficiency of the artificial compressibility method with a convective term and a diffusive term is investigated in Figure 13. The code has been executed on twelve Intel Xeon E5-2690V3 (144 CPU cores in total) using MPI parallel computing. Communication costs are minimal because of the explicit and local nature of the governing equations. Thus, the method becomes increasingly more time efficient than the projection method when the number of cores of the simulation is increased. 
Analysis of artificial pressure equations in numerical simulations of a turbulent channel flow

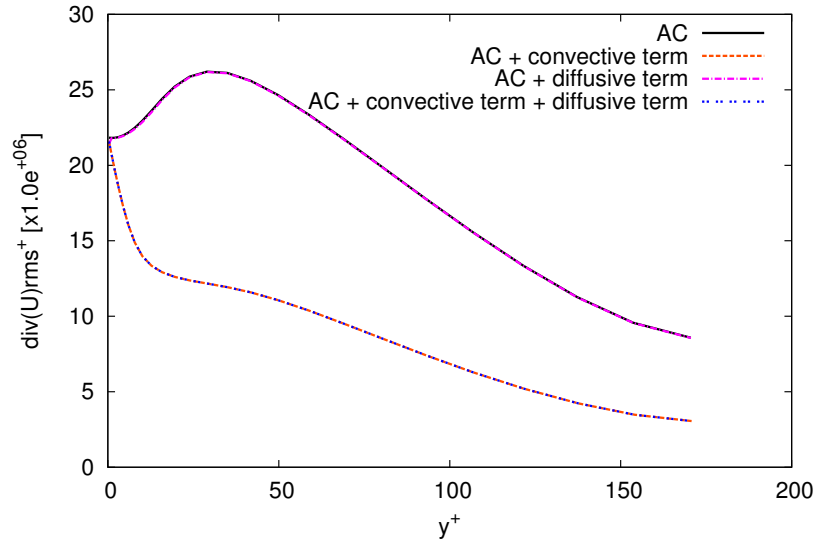

Figure 7 - Standard deviation of velocity divergence $\sqrt{\left\langle\left(\partial_{j} u_{j}^{\prime}\right)^{2}\right\rangle}$ for simulations on the coarse mesh at $M a=0.12$ using the artificial compressibility method (3), the artificial compressibility method with a convective term (18) and the artificial compressibility method with a diffusive term (17) and the artificial compressibility method with a convective and a diffusive term (16). 

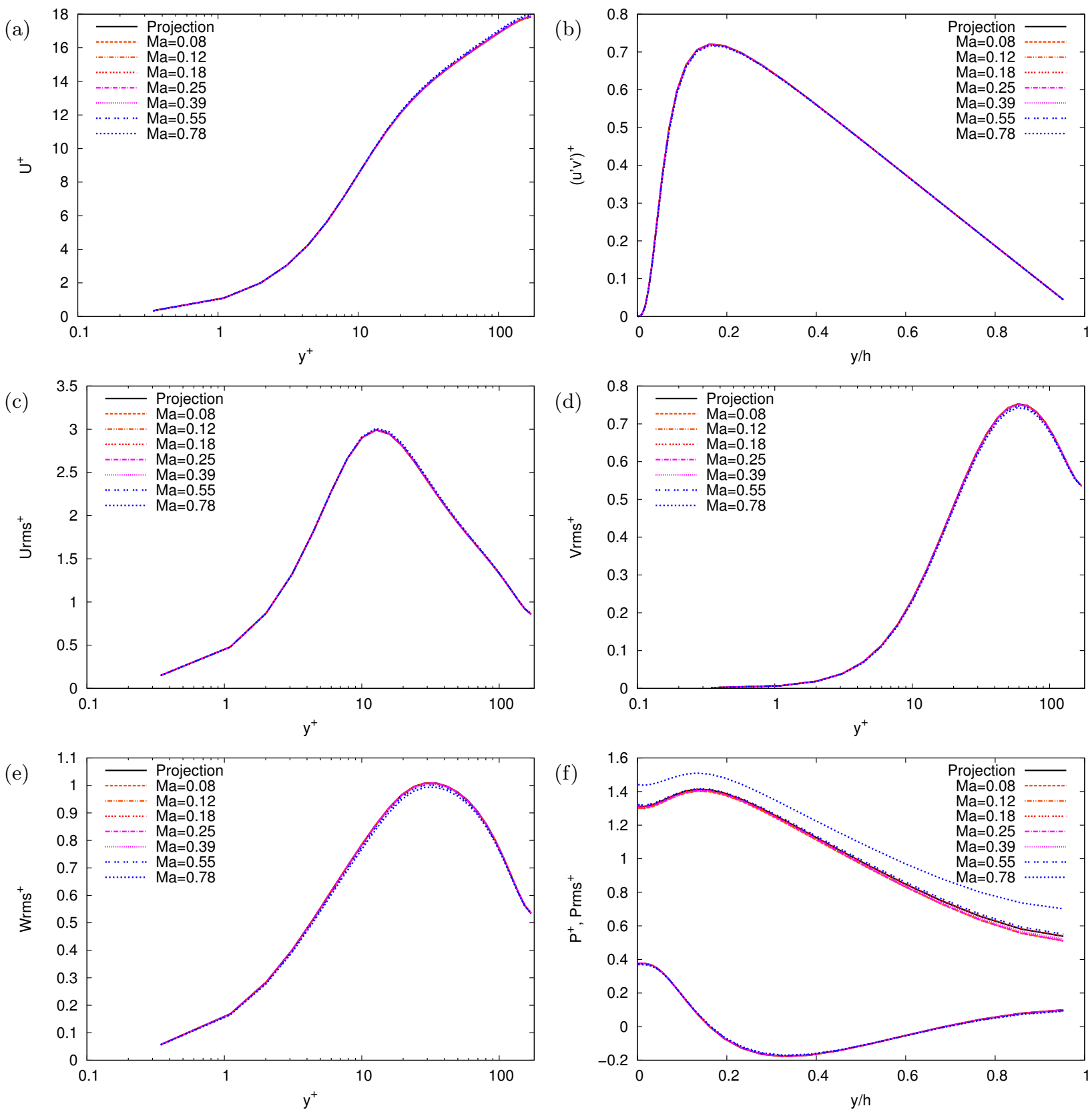

Figure 8 - Turbulence statistics for artificial compressibility simulations with a convective term on the coarse mesh and the corresponding simulation with a projection method. See Figure 3 for labels. 
Analysis of artificial pressure equations in numerical simulations of a turbulent channel flow
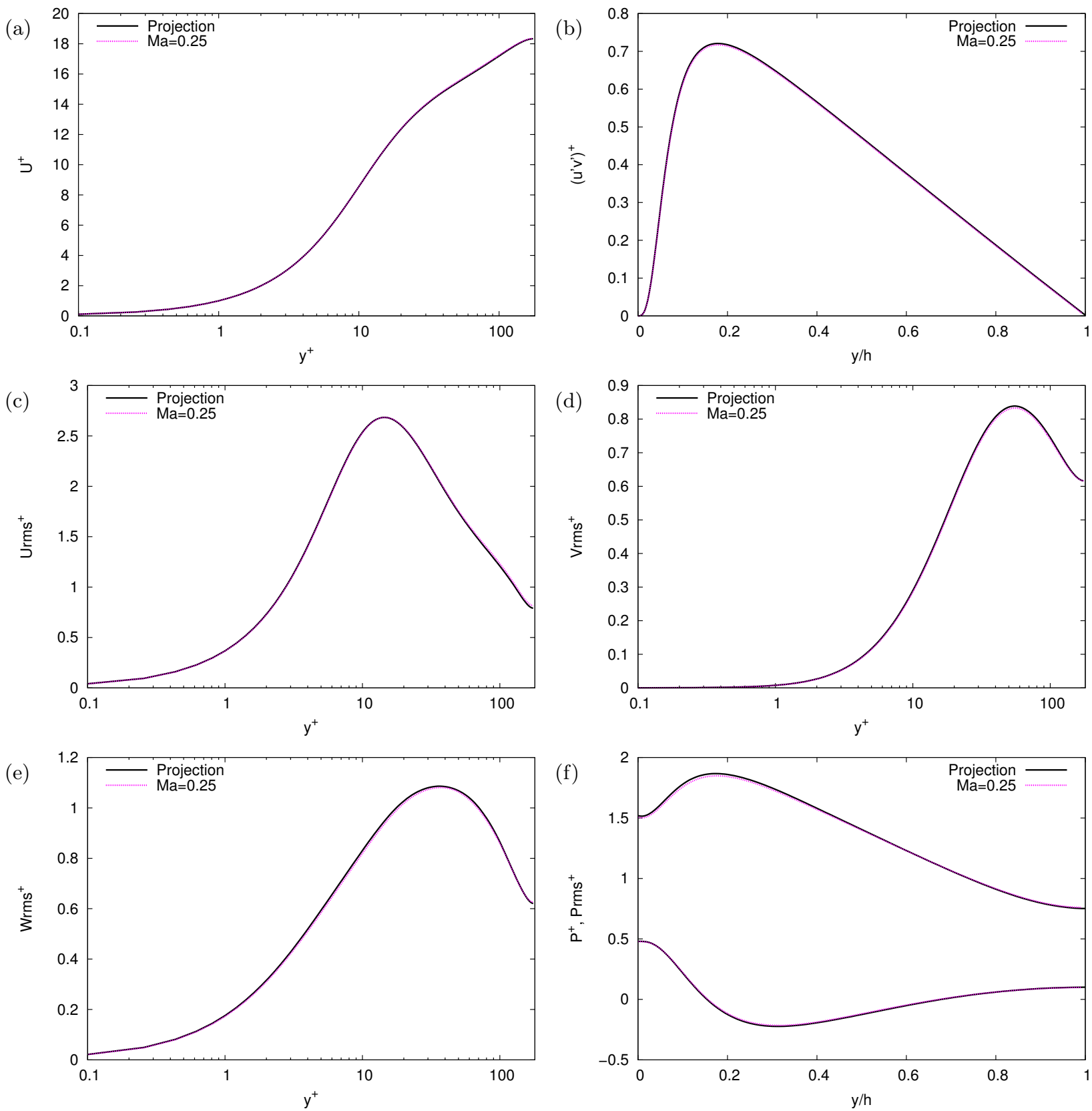

Figure 9 - Turbulence statistics for artificial compressibility simulations with a convective term on the fine mesh and the corresponding simulation with a projection method. See Figure 3 for labels. 

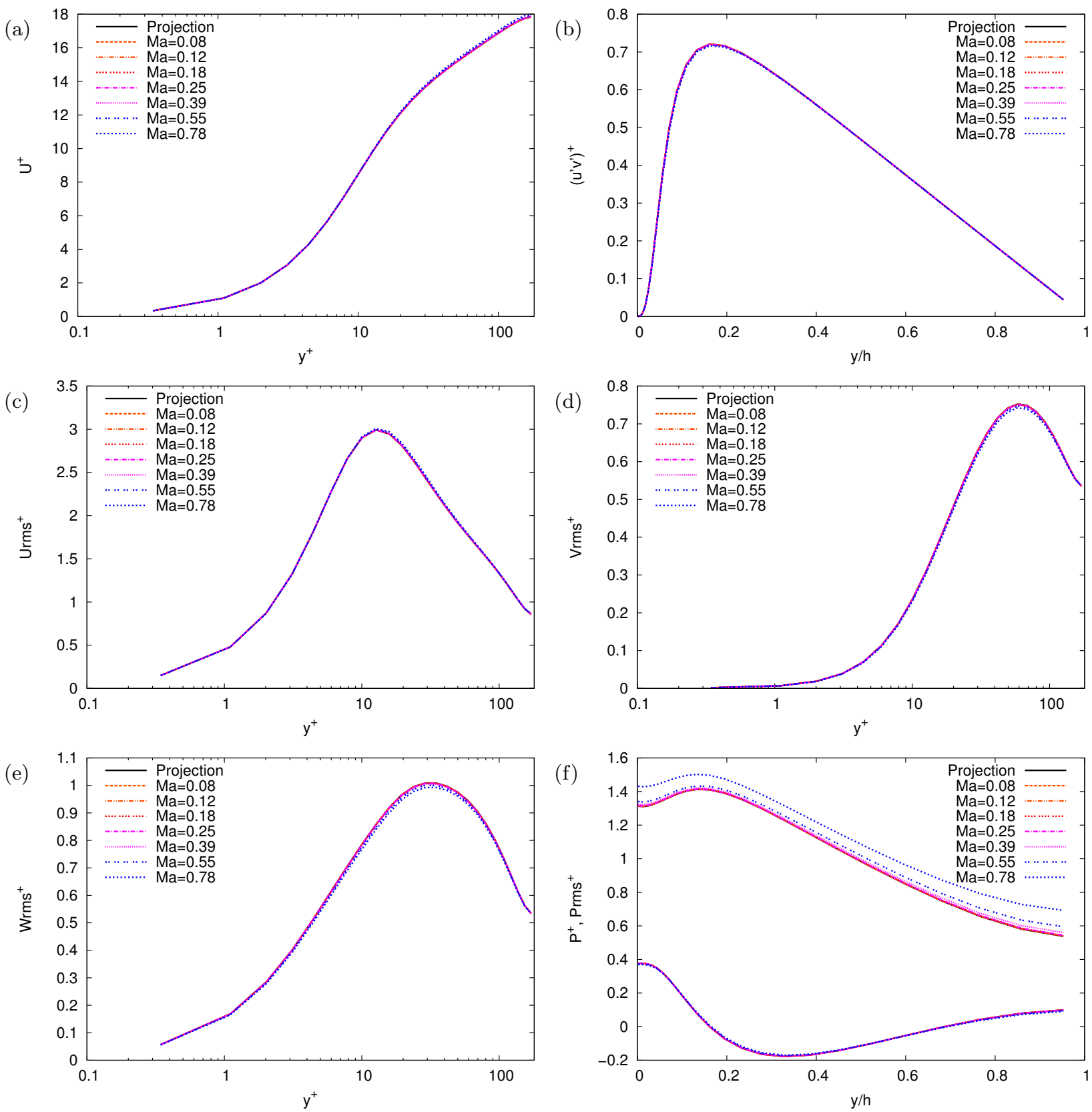

Figure 10 - Turbulence statistics for artificial compressibility simulations with a convective and a diffusive term on the coarse mesh and the corresponding simulation with a projection method. See Figure 3 for labels. 
Analysis of artificial pressure equations in numerical simulations of a turbulent channel flow
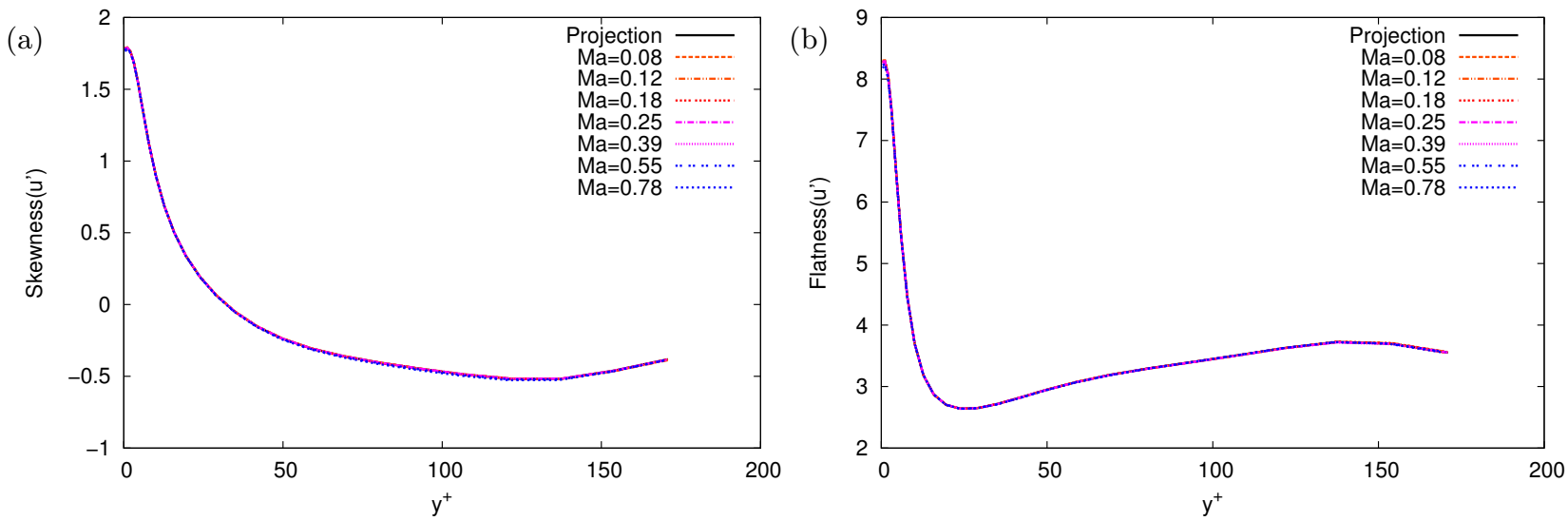

Figure 11 - Skewness (a) and flatness (b) factors of the longitudinal velocity for artificial compressibility simulations with a convective and a diffusive term on the coarse mesh and the corresponding simulation with a projection method. 

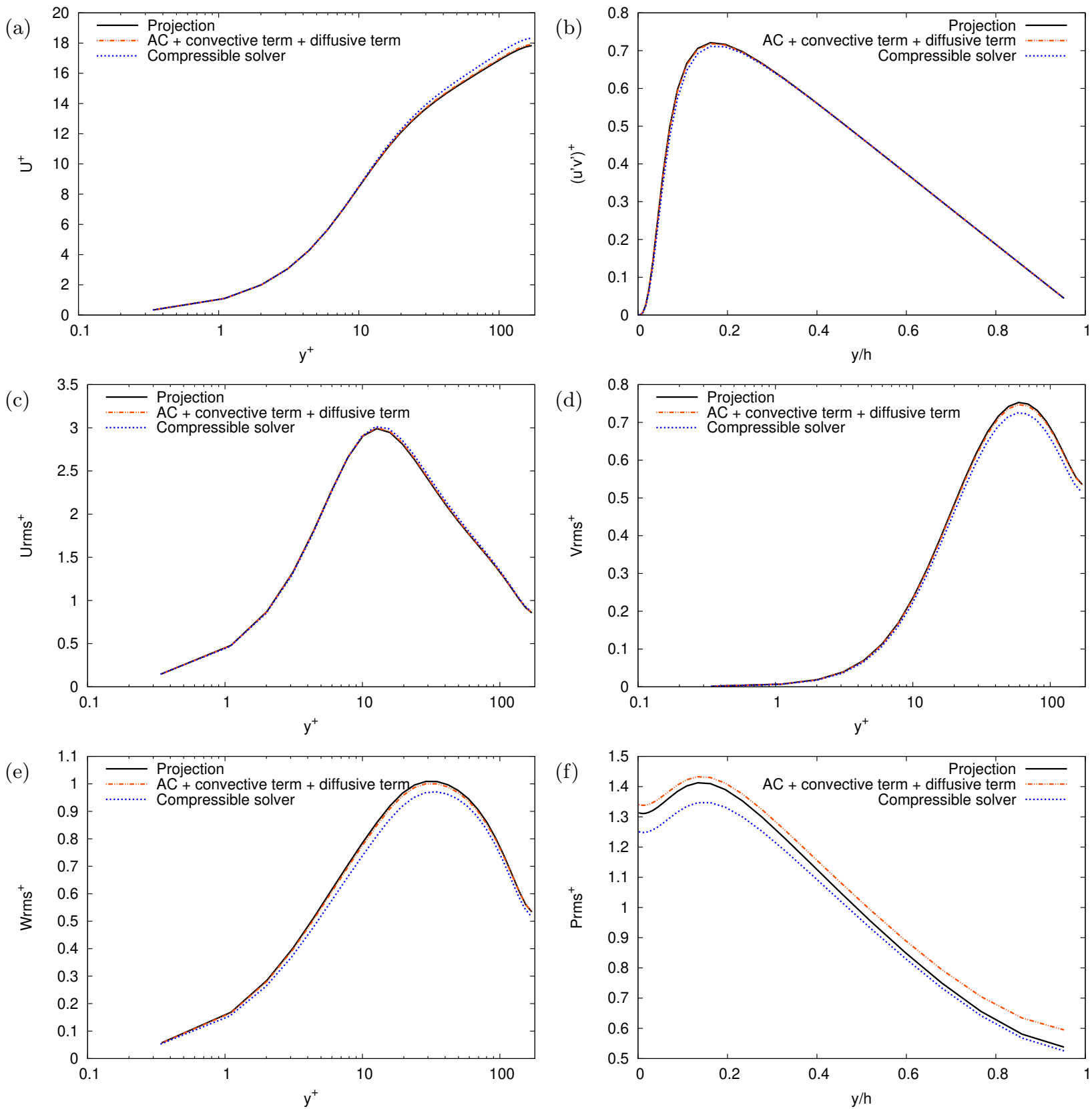

Figure 12 - Turbulence statistics for artificial compressibility simulations with a convective and a diffusive term on the coarse mesh compared to a compressible solver for the Mach number 0.55 . See Figure 3 for labels. 
Analysis of artificial pressure equations in numerical simulations of a turbulent channel flow

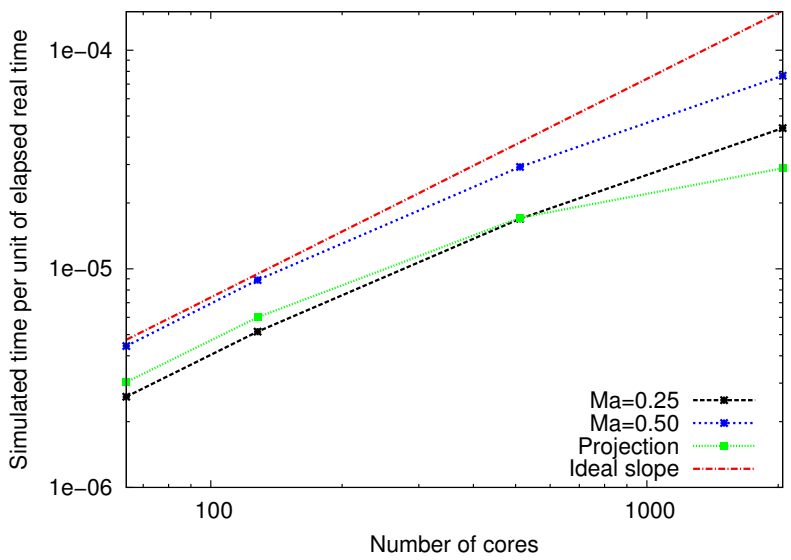

Figure 13 - Scalability of the artificial compressibility simulations with a convective and a diffusive term on the fine mesh and the corresponding simulation with a projection method. 


\section{Conclusion}

Simulations based on an artificial pressure equation produce velocity and pressure fields that can be made arbitrarily close to the velocity and pressure fields of the incompressible Navier-Stokes equations, at the cost of an increasingly large computational time. Several artificial pressure equations can be used depending on the selected level of approximation of the compressible Navier-Stokes equation. Amongst the methods examined in this paper, the artificial compressibility method with a convective and a diffusive term produces the most accurate results for a turbulent channel flow. The convective term is critical for the accuracy of the incompressibility constraint and all one-point turbulence statistics while the diffusive term only improves pressure fluctuations. The method is easily parallelisable because the system of equations is explicit in time and local in space, and has low memory requirements. The procedure is stable using a finite difference method in a staggered grid system and a third-order Runge-Kutta time scheme, even if no diffusive term is included, and can be used for underresolved simulations, large-eddy simulations or direct numerical simulations. We may conjecture that the method is near to optimal, in the sense that it is more effective than a compressible solver using a minimal amount of approximations on the one hand and simpler methods obtained by neglecting one by one the terms of the equation on the other hand. The approach can be combined with an extrapolation in terms of artificial Mach number, following the quadratic rate of convergence of the solution, in order to accelerate the rate of convergence.

\section{Acknowledgment}

This work was funded by the French Investments for the future ("Investissements d'Avenir") programme managed by the National Agency for Research (ANR) under contract ANR-10-LABX-22-01 (labex SOLSTICE). The authors gratefully acknowledge the CEA for the development of the TRUST platform. This work was granted access to the HPC resources of CINES under the allocations 2019-A0042A05099 made by GENCI.

\section{References}

[1] Y. Aghaee-Shalmani and H. Hakimzadeh. Numerical modeling of 2-d and 3-d flows using artificial compressibility method and collocated mesh. Journal of Applied Fluid Mechanics, 9 (5):2333-2345, 2016.

[2] T. Alazard. Low mach number limit of the full Navier-Stokes equations. Archive for Rational Mechanics and Analysis, 180(1):1-73, 2006.

[3] T. Alazard. A minicourse on the low mach number limit. Discrete and Continuous Dynamical Systems-Series S, 1(3):365-404, 2008.

[4] S. Ansumali, I. V. Karlin, and H. C. Öttinger. Thermodynamic theory of incompressible hydrodynamics. Physical Review Letters, 94(8):080602, 2005.

[5] P. Asinari, T. Ohwada, E. Chiavazzo, and A. F. Di Rienzo. Link-wise artificial compressibility method. Journal of Computational Physics, 231(15):5109-5143, 2012.

[6] R. Benzi, S. Succi, and M. Vergassola. The lattice boltzmann equation: theory and applications. Physics Reports, 222(3):145-197, 1992.

[7] P. Bigay, G. Oger, P.-M. Guilcher, and D. Le Touzé. A weakly-compressible cartesian grid approach for hydrodynamic flows. Computer Physics Communications, 220:31-43, 2017.

[8] I. A. Bolotnov, R. T. Lahey, D. A. Drew, K. E. Jansen, and A. A. Oberai. Spectral analysis of turbulence based on the DNS of a channel flow. Computers \& Fluids, 39(4):640-655, 2010. 
Analysis of artificial pressure equations in numerical simulations of a turbulent channel flow

[9] S. Borok, S. Ansumali, and I. V. Karlin. Kinetically reduced local navier-stokes equations for simulation of incompressible viscous flows. Physical Review E, 76(6):066704, 2007.

[10] C. Calvin, O. Cueto, and P. Emonot. An object-oriented approach to the design of fluid mechanics software. ESAIM: Mathematical Modelling and Numerical Analysis, 36(05):907921, 2002.

[11] D. Choi and C. L. Merkle. Application of time-iterative schemes to incompressible flow. AIAA Journal, 23(10):1518-1524, 1985.

[12] A. J. Chorin. A numerical method for solving incompressible viscous flow problems. Journal of Computational Physics, 2(1):12-26, 1967.

[13] A. J. Chorin. Numerical solution of the Navier-Stokes equations. Mathematics of Computation, 22(104):745-762, 1968.

[14] B. Christer and V. Johansson. The use of richardson extrapolation for the numerical solution of low mach number flow in confined regions. Journal of Scientific Computing, 8(4):307-340, 1993.

[15] J. R. Clausen. Entropically damped form of artificial compressibility for explicit simulation of incompressible flow. Physical Review E, 87(1):013309, 2013.

[16] Y. T. Delorme, K. Puri, J. Nordstrom, V. Linders, S. Dong, and S. H. Frankel. A simple and efficient incompressible navier-stokes solver for unsteady complex geometry flows on truncated domains. Computers $\mathscr{E}$ Fluids, 150:84-94, 2017.

[17] D. Dupuy, A. Toutant, and F. Bataille. Turbulence kinetic energy exchanges in flows with highly variable fluid properties. Journal of Fluid Mechanics, 834:5-54, 2018.

[18] D. Dupuy, A. Toutant, and F. Bataille. Effect of the reynolds number on turbulence kinetic energy exchanges in flows with highly variable fluid properties. Physics of Fluids, 31(1): 015104, 2019.

[19] D. Dupuy, A. Toutant, and F. Bataille. A posteriori tests of subgrid-scale models in an isothermal turbulent channel flow. Physics of Fluids, 31(4):045105, 2019.

[20] D. Dupuy, A. Toutant, and F. Bataille. A posteriori tests of subgrid-scale models in an anisothermal turbulent channel flow at low mach number. Physics of Fluids, 31(6):065113, 2019.

[21] M. Ellero and M. Tessarotto. An inverse kinetic theory for the incompressible navier-stokes equations. Physica A: Statistical Mechanics and its Applications, 355(2-4):233-250, 2005.

[22] J. Guerra and B. Gustafsson. A numerical method for incompressible and compressible flow problems with smooth solutions. Journal of Computational Physics, 63(2):377-397, 1986.

[23] B. Gustafsson and H. Stoor. Navier-stokes equations for almost incompressible flow. SIAM Journal on Numerical Analysis, 28(6):1523-1547, 1991.

[24] T. Hashimoto, I. Tanno, Y. Tanaka, K. Morinishi, and N. Satofuka. Simulation of doubly periodic shear layers using kinetically reduced local navier-stokes equations on a gpu. Computers G Fluids, 88:715-718, 2013.

[25] T. Hashimoto, I. Tanno, T. Yasuda, Y. Tanaka, K. Morinishi, and N. Satofuka. Higher order numerical simulation of unsteady viscous incompressible flows using kinetically reduced local navier-stokes equations on a gpu. Computers $\mathcal{E}$ Fluids, 110:108-113, 2015.

[26] T. Hashimoto, T. Yasuda, I. Tanno, Y. Tanaka, K. Morinishi, and N. Satofuka. Multi-gpu parallel computation of unsteady incompressible flows using kinetically reduced local navierstokes equations. Computers \& Fluids, 167:215-220, 2018.

[27] G. Hauke and T. J. R. Hughes. A unified approach to compressible and incompressible flows. Computer Methods in Applied Mechanics and Engineering, 113(3-4):389-395, 1994.

[28] X. He, G. D. Doolen, and T. Clark. Comparison of the lattice boltzmann method and the artificial compressibility method for navier-stokes equations. Journal of Computational Physics, 179(2):439-451, 2002.

[29] A. Kajzer and J. Pozorski. Application of the entropically damped artificial compressibility model to direct numerical simulation of turbulent channel flow. Computers $\&$ Mathematics with Applications, 76(5):997-1013, 2018.

[30] I. V. Karlin, A. G. Tomboulides, C. E. Frouzakis, and S. Ansumali. Kinetically reduced local navier-stokes equations: An alternative approach to hydrodynamics. Physical Review E, 74 (3):035702, 2006. 
[31] C. Kiris, J. Housman, and D. Kwak. Comparison of artificial compressibility methods. In Computational Fluid Dynamics 2004, pages 475-480. Springer, 2006.

[32] S. Klainerman and A. Majda. Singular limits of quasilinear hyperbolic systems with large parameters and the incompressible limit of compressible fluids. Communications on Pure and Applied Mathematics, 34(4):481-524, 1981.

[33] S. Klainerman and A. Majda. Compressible and incompressible fluids. Communications on Pure and Applied Mathematics, 35(5):629-651, 1982.

[34] H.-O. Kreiss, J. Lorenz, and M. J. Naughton. Convergence of the solutions of the compressible to the solutions of the incompressible navier-stokes equations. Advances in Applied Mathematics, 12(2):187-214, 1991.

[35] D. Kwak, J. L. C. Chang, S. P. Shanks, and S. R. Chakravarthy. A three-dimensional incompressible navier-stokes flow solver using primitive variables. AIAA Journal, 24(3):390-396, 1986.

[36] László L. Könözsy and Dimitris Drikakis. A unified fractional-step, artificial compressibility and pressure-projection formulation for solving the incompressible navier-stokes equations. Communications in Computational Physics, 16(5):1135-1180, 2014.

[37] M. Lee and R. D. Moser. Direct numerical simulation of turbulent channel flow up to $\operatorname{Re}_{\tau} \approx$ 5200. Journal of Fluid Mechanics, 774:395-415, 2015.

[38] B. P. Leonard. A stable and accurate convective modelling procedure based on quadratic upstream interpolation. Computer Methods in Applied Mechanics and Engineering, 19(1): 59-98, 1979.

[39] C. Liang, A. Chan, X. Liu, and A. Jameson. An artificial compressibility method for the spectral difference solution of unsteady incompressible navier-stokes equations on multiple grids. In 49th AIAA Aerospace Sciences Meeting including the New Horizons Forum and Aerospace Exposition, page 48, 2011.

[40] P.-L. Lions and T. H. Moulden. Mathematical topics in fluid mechanics, Volume 1: Incompressible models. Oxford University Press, New York, 1996.

[41] N. A. Loppi, F. D. Witherden, A. Jameson, and P. E. Vincent. A high-order cross-platform incompressible navier-stokes solver via artificial compressibility with application to a turbulent jet. Computer Physics Communications, 233:193-205, 2018.

[42] P. R. McHugh and J. D. Ramshaw. Damped artificial compressibility iteration scheme for implicit calculations of unsteady incompressible flow. International Journal for Numerical Methods in Fluids, 21(2):141-153, 1995.

[43] A. Meister. Asymptotic single and multiple scale expansions in the low mach number limit. SIAM Journal on Applied Mathematics, 60(1):256-271, 1999.

[44] C. L. Merkle and Y.-H. Choi. Computation of low-speed flow with heat addition. AIAA Journal, 25(6):831-838, 1987.

[45] C. L. Merkle and Y.-H. Choi. Computation of low-speed compressible flows with timemarching procedures. International Journal for Numerical Methods in Engineering, 25(2): 293-311, 1988.

[46] Y. Morinishi, T. S. Lundhomas, O. V. Vasilyev, and P. Moin. Fully conservative higher order finite difference schemes for incompressible flow. Journal of Computational Physics, 143(1): 90-124, 1998.

[47] R. D. Moser, J. Kim, and N. N. Mansour. Direct numerical simulation of turbulent channel flow up to $\operatorname{Re}_{\tau}=590$. Physics of Fluids, 11(4):943-945, 1999.

[48] F. Muldoon and S. Acharya. A modification of the artificial compressibility algorithm with improved convergence characteristics. International Journal for Numerical Methods in Fluids, 55(4):307-345, 2007.

[49] C. D. Munz, M. Dumbser, and M. Zucchini. The multiple pressure variables method for fluid dynamics and aeroacoustics at low mach numbers. Numerical Methods for Hyperbolic and Kinetic Problems, 7:335-359, 2003.

[50] C.-D. Munz, S. Roller, R. Klein, and K. J. Geratz. The extension of incompressible flow solvers to the weakly compressible regime. Computers \& Fluids, 32(2):173-196, 2003.

[51] F. Nicoud. Conservative high-order finite-difference schemes for low-Mach number flows. Journal of Computational Physics, 158(1):71-97, 2000. 
[52] T. Ohwada and P. Asinari. Artificial compressibility method revisited: asymptotic numerical method for incompressible navier-stokes equations. Journal of Computational Physics, 229 (5):1698-1723, 2010.

[53] T. Ohwada, P. Asinari, and S. Yabusaki. Artificial compressibility method and lattice boltzmann method: Similarities and differences. Computers \& Mathematics with Applications, 61 (12):3461-3474, 2011

[54] P. J. O'Rourke and F. V. Bracco. Two scaling transformations for the numerical computation of multidimensional unsteady laminar flames. Journal of Computational Physics, 33(2):185203, 1979.

[55] J. D. Ramshaw, P. J. O'Rourke, and L. R. Stein. Pressure gradient scaling method for fluid flow with nearly uniform pressure. Journal of Computational Physics, 58(3):361-376, 1985.

[56] M. Salinas-Vázquez, W. Vicente, E. Barrios, E. Martínez, A. Palacio, and A. Rodríguez. A low-mach number method for the numerical simulation of complex flows. Applied Mathematical Modelling, 37(22):9132-9146, 2013.

[57] J. Shen. Pseudo-compressibility methods for the unsteady incompressible navier-stokes equations. In Proceedings of the 1994 Beijing symposium on nonlinear evolution equations and infinite dynamical systems, pages 68-78, 1997.

[58] R. Temam. Sur l'approximation de la solution des équations de Navier-Stokes par la méthode des pas fractionnaires (ii). Archive for Rational Mechanics and Analysis, 33(5):377-385, 1969.

[59] M. Tessarotto, M. Ellero, N. Aslan, M. Mond, and P. Nicolini. Exact pressure evolution equation for incompressible fluids. In AIP Conference Proceedings, volume 1084, pages 224229. AIP, 2008.

[60] A. Toutant. General and exact pressure evolution equation. Physics Letters A, 381(44): $3739-3742,2017$.

[61] A. Toutant. Numerical simulations of unsteady viscous incompressible flows using general pressure equation. Journal of Computational Physics, 374:822-842, 2018.

[62] A. W. Vreman and J. G. M. Kuerten. Comparison of direct numerical simulation databases of turbulent channel flow at $\mathrm{Re}_{\tau}=180$. Physics of Fluids, 26(1):015102, 2014.

[63] Y. Wang and A. Trouvé. Artificial acoustic stiffness reduction in fully compressible, direct numerical simulation of combustion. Combustion Theory and Modelling, 8(3):633-660, 2004.

[64] J. H. Williamson. Low-storage Runge-Kutta schemes. Journal of Computational Physics, 35 (1):48-56, 1980 . 